\title{
Acute Epidural Hematoma Secondary to Skull Metastasis from Esophageal Carcinoma
}

\author{
Michael J. Ellis, Patrick J. McDonald
}

Can. J. Neurol. Sci. 2007; 34: 491-493

The skull may be a site of metastasis in malignancies such as carcinoma of the breast, prostate, lung, and multiple myeloma. ${ }^{1}$ The majority of patients with skull metastases are asymptomatic or present with localized swelling which is often painless. ${ }^{2}$ While subdural hematoma has been commonly associated with metastatic disease involving the dura, ${ }^{3}$ hemorrhage from a skull metastasis remains a rare occurrence. We present the case of acute epidural hematoma resulting from metastatic esophageal carcinoma to the skull.

\section{Case Report}

A 41-year-old man was found at home unresponsive. Examination in the emergency room showed left-sided hemiparesis and a Glasgow Coma Score (GCS) of 12/15 (Motor 6 , Verbal 3, Eye opening 3) with a normal cranial nerve exam and equal and reactive pupils. The patient had a one-year history of esophageal carcinoma treated with thoracoabdominal esophagogastrectomy as well as radiation and chemotherapy. He was felt to be free of metastatic disease.

The patient underwent a computed tomograpy (CT) scan of the head which identified a right parietal-occipital lytic bone lesion and a $6 \mathrm{~cm}$ epidural hematoma, with compression of the right lateral ventricle and midline shift (Figure).

The patient's level of consciousness (LOC) deteriorated to a GCS of $8 / 15$ requiring intubation for airway protection and urgent surgery. After opening the scalp and exposing the skull, a large lytic bone lesion was encountered along the medial aspect of the occipital bone. The tumor had eroded through the skull and clotted blood was found extruding through the bone itself. An elliptical craniotomy was fashioned extending from the lytic lesion to a burr hole placed just anterior to the squamosal suture. After removal of the bone flap, a large epidural hematoma was evacuated. We identified and resected an area of dura that had been invaded by the lytic bone tumor which appeared to be the source of hemorrhage. Working medially from the margin of the bone flap, we removed tumor-infiltrated bone until solid normal appearing bone was encountered. Because of the relatively small amount of bone removed, a cranioplasty was not undertaken. Bone and soft tissue samples were sent for pathological examination.

Post-operatively the patient's LOC improved immediately and his left-sided hemiparesis resolved over 24 hours. A follow-up CT scan taken four days post-operatively showed complete resolution of the epidural hematoma. The patient was discharged four days after surgery on a tapering dose of dexamethasone.
Pathologic examination of intraoperative specimens revealed metastatic involvement of the skull as well as diffuse invasion of the meninges. The patient underwent local radiotherapy to the skull area but ultimately succumbed to his primary disease 11 months after surgery, without recurrence of any neurologic symptoms.

\section{DisCUSSION}

Esophageal carcinoma is an aggressive malignancy, which frequently spreads to the lymph nodes, liver, lung, adrenals, airways, stomach, kidney, and bone. ${ }^{4}$ Although metastatic involvement of the skull has been observed in carcinoma of the breast, prostate, and lung among others, ${ }^{1}$ skull metastasis arising from esophageal carcinoma is exceedingly rare. ${ }^{5}$ In addition, acute hemorrhage secondary to skull metastases has rarely been described. Acute epidural hematoma resulting from skull metastases has been reported in only three cases.

These cases include two of metastatic hepatocellular carcinoma and one of small cell carcinoma of the lung presenting with skull metastases and epidural hematoma. ${ }^{6-8}$ All required evacuation of the hematoma with improvement in symptoms. All patients eventually succumbed to their primary disease.

To our knowledge, our case represents the fourth reported case of acute epidural hematoma secondary to skull metastasis and the only case of epidural hematoma secondary to esophageal carcinoma metastasis to the skull, although one case of cervical esophageal cancer metastasizing to the temporal bone has been reported. ${ }^{5}$

In the present case, the mechanism of spread from the esophagus and affected lymph nodes to the skull is unclear. Hematogenous spread from the primary lesion, perineural or perivascular spread through skull foramina, ${ }^{9}$ as well as invasion through the skull sutures ${ }^{10}$ have all been proposed to explain the

From the Section of Neurosurgery, Department of Surgery, University of Manitoba, Winnipeg, Manitoba, Canada

ReCEIVED SePtember 29, 2006. ACCEPTED IN FInAL FORm June 11, 2007. Reprint requests to: Patrick J. McDonald, Section of Neurosurgery, Department of Surgery, University of Manitoba, Health Sciences Centre, GB126- 820 Sherbrooke Street, Winnipeg, Manitoba, R3A 1R9, Canada. 


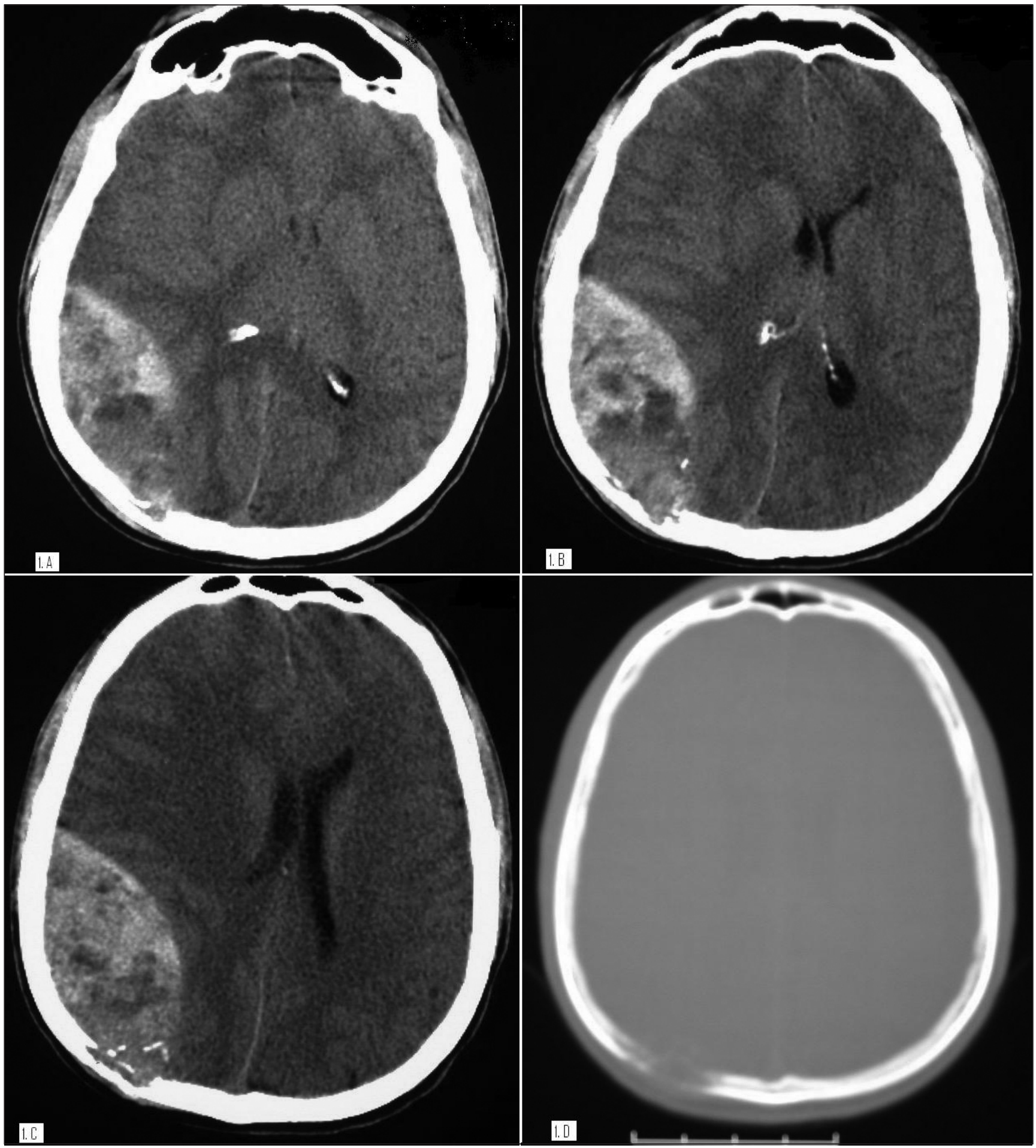

Figure: (A-D): Axial computerized tomography $(C T)$ scan demonstrating a right parietal-occipital lytic bone lesion and large epidural hematoma measuring $6 \mathrm{~cm}$, with compression of the right lateral ventricle and midline shift. Increased signal attenuation is seen within the lentiform mass consistent with acute hemorrhage. Figure $(D)$ demonstrates the lytic skull metastasis on bone windows. 
routes of metastatic spread to the skull. Given that patients with carcinoma of the lower esophagus rarely demonstrate metastasis to cervical nodes ${ }^{11}$ where they can infiltrate nerves and vessels ascending into the brain, hematogenous seeding of the calvarium may appear to be a more plausible mechanism in this case. Indeed esophageal carcinoma has been reported to metastasize to the brain ${ }^{12}$ and appears to do so through hematogenous spread via the arterial circulation. ${ }^{13}$

Although subdural hematoma has been described in cases of dural metastasis arising from of a number of primary malignancies, ${ }^{3}$ epidural hematoma secondary to skull metastasis is rare. Hemorrhage from a skull tumor may potentially arise from invasion of emissary veins, venous sinuses, or the middle meningeal artery, or may arise directly from the tumor itself. In this case, we suspect that hemorrhage occurred into the epidural space from the metastatic tumor as well as from the diploe of the invaded skull.

Given the rarity of this clinical presentation, meaningful recommendations are difficult to make. Metastatic skull lesions alone are relatively more common and efforts have been made to establish the indications for operating on these patients. Wecht and Sawaya ${ }^{14}$ suggest that the criteria for operating on patients with calvarial lesions should include: 1) the absence of a definitive diagnosis 2) if the lesion can be removed safely 3 ) if the lesion constitutes the only remaining residue of a documented malignancy in remission and 4) if the lesion appears symptomatic or is refractory to conventional radiation and chemotherapy. In our experience, we would add that the presence of lytic skull lesions poses a risk of intracranial invasion and resultant hemorrhage. Given the rarity of spontaneous hemorrhage from a skull metastasis, their presence alone does not constitute an indication for surgery.

\section{REFERENCES}

1. Maroldi R, Ambrosi C, Farina D. Metastatic disease of the brain: extra-axial metastases (skull, dura, leptomeningeal) and tumour spread. Eur Radiol. 2005;15(3):617-26.

2. Stark AM, Eichmann T, Mehdorn M. Skull metastases: clinical features, differential diagnosis, and review of the literature. Surg Neurol. 2003;60(3):219-25.

3. Laigle-Donadey F, Taillibert S, Mokhtari K, Hildebrand J, Delattre JV. Dural metastases. J Neurooncol. 2005;75(1):57-61.

4. Anderson LL, Lad TE. Autopsy findings in squamous-cell carcinoma of the esophagus. Cancer. 1982;50(8):1587-90.

5. Imauchi Y, Kaga K, Nibu K, Sakuma N, Iino Y, Kodera K. Metastasis of cervical esophageal carcinoma to the temporal bone- a study of the temporal bone histology. Auris Nasus Larynx. 2001;28(2):169-72.

6. Hayashi K, Matsuo T, Kurihara M, Daikoku M, Kitange G, Shibata S. Skull metastasis of hepatocellular carcinoma associated with acute epidural hematoma: a case report. Surg Neurol. 2000;53(4):379-82.

7. Nakagawa Y, Yoshino E, Suzuki K, Tatebe A, Andachi H. Spontaneous epidural hematoma from a hepatocellular carcinoma metastasis to the skull-case report. Neurol Med Chir (Tokyo). 1992;32(5):300-2.

8. Simmons NE, Elias WJ, Henson SL. Small cell lung carcinoma causing epidural hematoma: case report. Surg Neurol. 1999;51(1):56-9.

9. Jahn AF, Farkashidy J, Berman JM. Metastatic tumors in the temporal bone- a pathophysiologic study. J Otolaryngol. 1979;8(1):85-95.

10. Nakamura M, Kaga K, Ohira Y. Metastatic hypopharyngeal carcinoma to the temporal bone. Eur Arch Otohinolaryngol. 1996;253(3):185-8.

11. Sharma S, Fujita H, Yamana H, Kakegawa T. Patterns of lymph node metastasis in 3-field dissection for carcinoma in the thoracic esophagus. Surg Today. 1994;24(5):410-4.

12. Weinberg JS, Suki D, Hanbali F, Cohen ZR, Lenzi R, Sawaya R. Metastasis of esophageal carcinoma to the brain. Cancer. 2003;98(9):1925-33.

13. Ogawa K, Toita T, Sueyama H, Fuwa N, Kakinohana Y, Kamata M, et al. Brain metastases from esophageal carcinoma. Natural history, prognostic factors, and outcome. Cancer. 2002; 94(3):759-64.

14. Wecht DA, Sawaya R. Lesions of the calvaria: surgical experience with 42 patients. Ann Surg Oncol. 1997;4(1):28-36. 